\title{
具有一维链状金属原子间相互作用的 $\mathrm{Au}(\mathrm{I})$ 化合物 $\left[\mathrm{AuS}_{2} \mathrm{CNEt}_{2}\right]_{4 n}$ 的合成和 晶体结构及性质*
}

\author{
洪茂椿雷新建黄致盈康北笙江飞龙 刘汉钦 \\ (中国科学院福建吻质结构研究所, 结构化学开放研究实验室, 福州 350002)
}

\section{关键词 $\mathrm{Au}(\mathrm{I})-\mathrm{S}_{2} \mathrm{CNEt}_{2}$ 化合物、一维链状金属原子间相互作用、晶体结构}

含有二硫代氨基甲酸根 $\left(\mathrm{R}_{2} \mathrm{dtc}\right)$ 配位的低价硬币金属 $(\mathrm{Cu} 、 \mathrm{Ag} 、 \mathrm{Au} 、 \mathrm{Ni} 、 \mathrm{Pt})$ 化合物的报道屡 见不鲜 ${ }^{[1-3]}$. 然而, 金属原子以一维链状排列且具有 $\mathrm{M}-\mathrm{M}$ 间相互作用的硬币金属化合物却很 少见 ${ }^{[4]}$. 我们在研究低价铜和二硫代氨基甲酸根配体的化合物中得到一系列异金属的原子簇 化合物 ${ }^{[5-7]}$, 这里报道的是在 $\mathrm{Au}(\mathrm{I})-\mathrm{R}_{2} \mathrm{dtc}$ 体系中所得到的一种一维链状且具有较强的金属 原子间相互作用的化合物 $\left[\mathrm{AuS}_{2} \mathrm{CNEt}_{2}\right]_{4 n}$ 的合成、晶体结构和谱学性质.

\section{一、实验}

1. 化合物的合成 $\mathrm{Ph}_{3} \mathrm{PAuCl}$ 由摩尔数比为 $1: 2$ 的 $\mathrm{HAuCl}_{4} \cdot 3 \mathrm{H}_{2} \mathrm{O}$ 和 $\mathrm{Ph}_{3} \mathrm{P}$ 在乙醇介 质中反应制得. 所得到的 $\mathrm{Ph}_{3} \mathrm{PAuCl}(1.00 \mathrm{~g}, 2 \mathrm{mmol})$ 和 $\mathrm{NaS}_{2} \mathrm{CNEt}_{2} \cdot 3 \mathrm{H}_{2} \mathrm{O}(0.90 \mathrm{~g}, 4 \mathrm{mmol})$ 混和于 $100 \mathrm{ml}$ 的 $\mathrm{EtOH} / \mathrm{CH}_{2} \mathrm{Cl}_{2}(V: V-1: 1)$ 溶液中室温下搅拌 $24 \mathrm{~h}$, 溶液逐渐变成桔红色. 过滤后在室温下静置蒸发, 数日后析出桔红色柱状晶体. $1 \mathrm{R}$ 谱的主要吸收峰为: $280 \mathrm{~m}$, $445 \mathrm{~m}, 554 \mathrm{~s}, 658 \mathrm{~s}, 834 \mathrm{~s}, 980 \mathrm{~s}, 1200 \mathrm{vs}, 1416 \mathrm{vscm}^{-1}$.

2. 晶体结构测定选取尺寸为 $0.2 \times 0.2 \times 0.4 \mathrm{~mm}$ 的单晶装在 RIGAKU AFC5R 四圆 衍射仪上以 $\mathrm{MoK} \alpha$ 射线, $\theta-2 \theta$ 当描方式收集衍射数据. 晶体属于正交晶系, 空间群为 $\mathrm{Fddd}$, $a=26.471(7), b \doteq 45.160(11), c=24.661$ (7) $\AA$; $V=29480.5 \AA^{3}, D_{c}=2.490 \mathrm{gcm}^{-3}$, $z=32$. 在 $2^{\circ} \leqslant \theta \leqslant 50^{\circ}$ 范围内共收集 7650 独立的衍射点, 其中 3095 个 $I \geqslant 3 \sigma(I)$ 衍射 点用于结构分析. 以直接法和差 Fourier 合成获得全部非氢原子的坐标, 氢原子坐标按理论 模型求算但不参加结构的修正. 以全矩阵最小二乘方对所有的非氢原子的坐标及各向异性温 度因子修正,最终偏离因子为: $R=0.058, R_{w}=0.062$.

3. 物理方法测试红外光謟在 Perkin-Elmer-577 型红外谱仪上测定, KBr 压片. 磁化 率在本所自制的古依-法拉弟两用磁天平上由法拉弟法测得. ${ }^{1} \mathrm{H} N M R$ 谱图在 Varian-FT $80 \mathrm{~A}$ 核磁共振谱仪上测定。

1991-03-08 收稿, 1992-04-06 收修改稿

* 国家自然科学幕金资助项目 
表 1 化合物 $\left[\mathrm{AuS}_{2} \mathrm{CNEr}_{2}\right]_{4 \text { n }}$ 的主要键长 $(\AA)$ 和键角值 $\left(^{\circ}\right)$

\begin{tabular}{|c|c|c|c|}
\hline$A u(1)-A u(1)^{\prime}$ & $3.037(1)$ & $A u(1)-A u(2)$ & $2.775(1)$ \\
\hline$A u(2)-A u(3)$ & $2.985(1)$ & $A u(3)-A u(4)$ & $2.761(1)$ \\
\hline$A u(4)-A u(4)^{\prime}$ & $2.981(1)$ & & \\
\hline $\mathrm{Au}(1)-\mathrm{S}(1)$ & $2.273(4)$ & $A u(1)-S(2)$ & $2.312(4)$ \\
\hline$A u(2)-S(3)$ & $2.275(5)$ & $A u(2)-S(4)$ & $2.270(4)$ \\
\hline $\mathrm{Au}(3)-S(5)$ & $2.313(5)$ & $\mathrm{Au}(3)-\mathrm{S}(6)$ & $2.287(6)$ \\
\hline $\mathrm{Au}(4)-S(7)$ & $2.282(5)$ & $A u(4)-S(8)$ & $2.284(5)$ \\
\hline$A u(1)^{\prime}-A u(1)-A u(2)$ & $177.89(5)$ & $A u(1)-A u(2)-A u(3)$ & $168.85(5)$ \\
\hline$A u(2)-A u(3 ;-A u(4)$ & $171.68(6)$ & $A u(4)^{\prime}-A u(4)-A u(3)$ & $161.55(6)$ \\
\hline$A u(1)^{\prime}-A u(1)-S(1)$ & $90.4(2)$ & $A u(1)^{\prime}-A u(1)-S(2)$ & $84.9(2)$ \\
\hline$A u(2)-A u(1)-S(1)$ & $91.4(2)$ & $A u(2)-A u(1)-S(2)$ & $93.4(2)$ \\
\hline$A u(1)-A u(2)-S(3)$ & $94.0(2)$ & $A u(1)-A u(2)-S(4)$ & $91.6(2)$ \\
\hline$A u(3)-A u(2)-S(3)$ & $90.1(2)$ & $A u(3)-A u(2)-S(4)$ & $84.5(3)$ \\
\hline$A u(2)-A u(3)-S(5)$ & $86.7(3)$ & $A u(2)-A u(3)-S(6)$ & $92.2(2)$ \\
\hline$A u(4)-A u(3)-S(5)$ & $91.4(3)$ & $\mathrm{Au}(4)-\mathrm{Au}(3)-\mathrm{S}(6)$ & $89.8(3)$ \\
\hline$A u(3)-A u(4)-S(7)$ & $90.6(2)$ & $\mathrm{Au}(3)-\mathrm{Au}(4)-\mathrm{S}(8)$ & $90.4(2)$ \\
\hline $\mathrm{Au}(4)^{\prime}-\mathrm{Au}(4)-\mathrm{S}(7)$ & $88.9(2)$ & $\mathrm{Au}(4)^{\prime}-\mathrm{Au}(4)-\mathrm{S}(8)$ & $91.3(2)$ \\
\hline$S(1)-A u(1)-S(2)$ & $175.2(3)$ & $S(3)-A u(2)-S(4)$ & $174.6(3)$ \\
\hline$S(5)-A u(3)-S(6)$ & $178.8(3)$ & $S(7)-A u(4)-S(8)$ & $176,3(3)$ \\
\hline
\end{tabular}

\section{二、结果和讨论}

$X$-射线单晶衍射分析的结果, 按结晶学描述, 独立的原子组成了四聚单元, $\left[\mathrm{AuS}_{2} \mathrm{CNEt}_{2}\right]_{4}$.

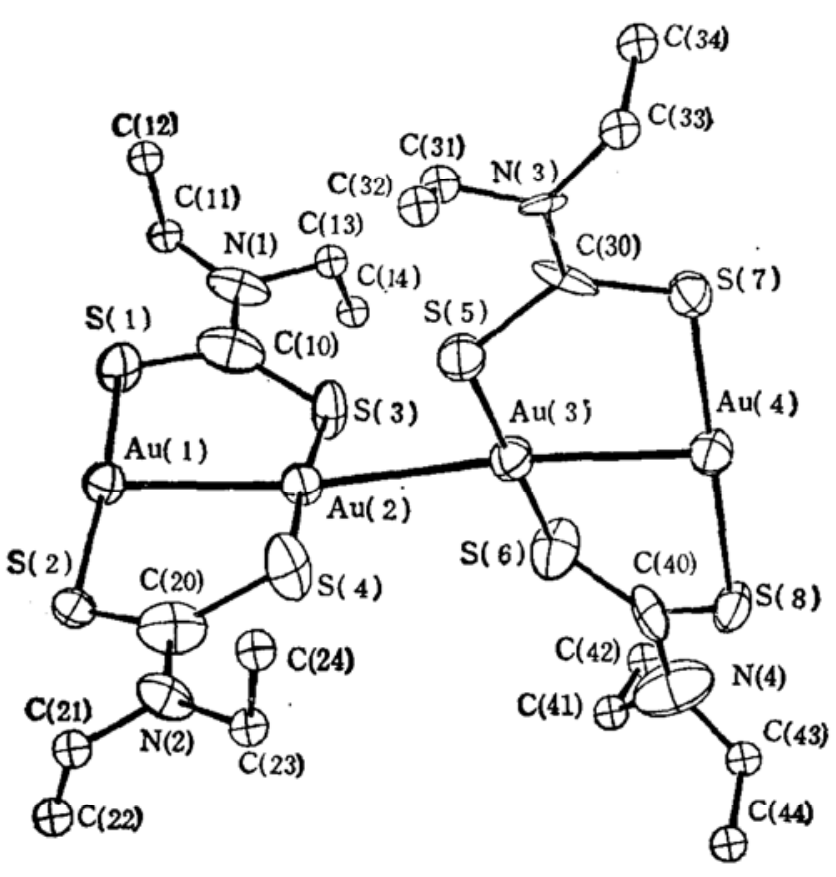

图 1 四聚单元 $\left[\mathrm{AuS}_{2} \mathrm{CNEt}_{2}\right]$ ，的结构 在单胞中, 这些四聚单元通过 $A u-A u$ 相 互作用连成近似于直线的一维 链状 结 构, $\left[\mathrm{AuS}_{2} \mathrm{CNEt}_{2}\right]_{4 n}$. 四聚单元及省略去 C、 $N$ 原子的单咆内分子堆积状态分别 示于图 1 和图 2.

从图 1 可见，独立的原子中每两个 金原子和两个 $\mathrm{Et}_{2} \mathrm{dtc}$ 配体以硫原子为配 位端基，鳌合成一个近似于平面的八圆 环. 金原子与两个来自不同的 $\mathrm{R}_{2} \mathrm{dtc}$ 配 体的硫原子配位, 配位构形为直线型, S-Au-S 键角落在 $174-179^{\circ}$ 之间. 两 个这样的八圆环又以平面交叉约为 $90^{\circ}$ 并通过 $\mathrm{Au}-\mathrm{Au}$ 相互作用结合在一起, 形成四聚的独立单元.

在这一维链状结构中, 金原子间距 分成长短两类. 其一为八圆环内的金原 子间距,其距离较短,落在 $2.761-2.775 \AA$ 之间; 其二为毗邻八圆环之间, $\mathrm{Au}-\mathrm{Au}$ 落在 2.985$3.037 \AA$ 之间. 整体的 $\mathrm{Au}-\mathrm{Au}$ 平均间距为 $2.908 \AA$, 类似于 $\left[\mathrm{AuS}_{2} \mathrm{CNEt}_{2}\right]_{2}$ 的 $\mathrm{Au}-\mathrm{Au}$ 平均距 离 ${ }^{[4]}$, 但比其他的 $\mathrm{Au}(\mathrm{I})$ 化合物的 $\mathrm{Au}-\mathrm{Au}$ 距离短得多 $\mathrm{Au}_{2}(\mathrm{tmb}) \mathrm{Cl}_{2}, 3.306 \AA^{[8]} ; \mathrm{K}\left[\mathrm{Au}(\mathrm{CN})_{2}\right]$, 


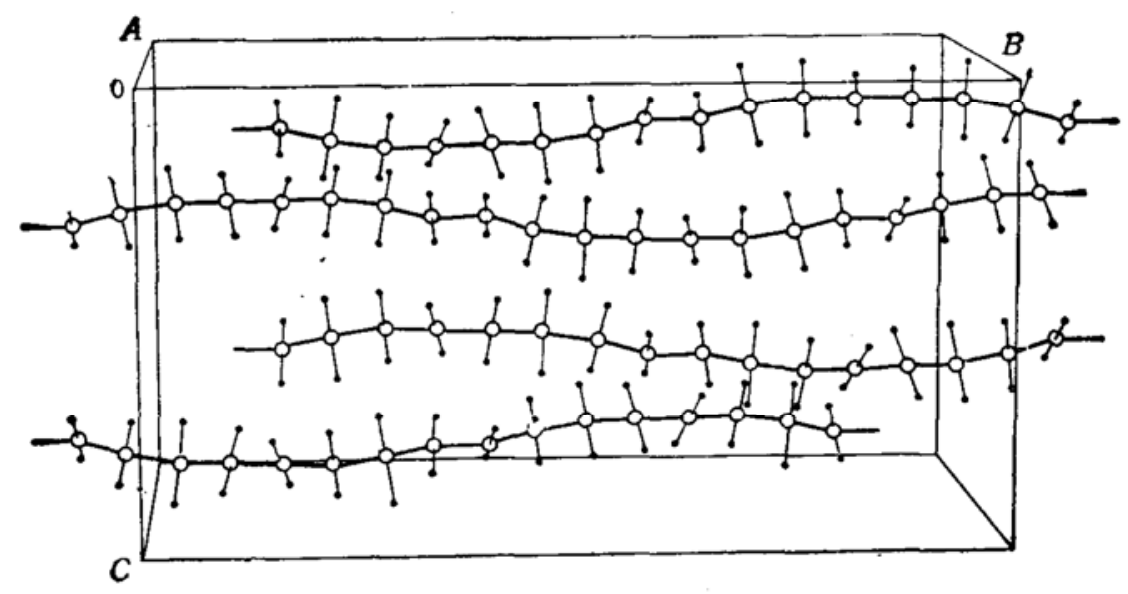

图 $2\left[\mathrm{AuS}_{2} \mathrm{CNEt}_{2}\right]_{4 n}$ 的单胞堆积图

$\left.3.64 \AA^{[9]} ; \mathrm{Au}_{2}(\mathrm{dmb}) \mathrm{CN}, 3.536 \AA^{[0]}\right)$. 表 1 列出重要的键长、键角的值. 本文报道的化合物的 最主要的结构特征是具有较短的 $\mathrm{Au}-\mathrm{Au}$ 距离和一维链状的金属原子定向排布 $(\mathrm{Au}-\mathrm{Au}-\mathrm{Au}$ 键 角落在 $162-179^{\circ}$ 之间),金原子之间存在着较强的相互作用. 这种结构的晶体可能具有特殊 的电导性能, 有关的电学特性正在研究之中.

磁化率测定表明该化合物为抗磁性. 二乙基二硫代氨基甲酸根配体 中的 $\mathrm{CH}_{3} \mathrm{CH}_{2}$ 一的 ${ }^{1} \mathrm{HNMR}$ 讯号末见顺磁加宽. 在 $\mathrm{CD}_{2} \mathrm{Cl}_{2}$ 溶液中 $\mathrm{CH}_{3}$ 一和 $-\mathrm{CH}_{2}$ 一的化学位移分别为 1.33 和 $3.96 \mathrm{ppm}$. 化合物的 IR 谱比较复杂, 其中 280,980 和 $1416 \mathrm{~cm}^{-1}$ 的三个吸收峰分别被指认为 $\mathrm{Au}-\mathrm{S}, \mathrm{C}-\mathrm{S}$ 和 $\mathrm{C}-\mathrm{N}$ 的伸缩振动.

\section{参 考 文 献}

[1] Anacker-Eickhoff, H., Hesse, R., Jennische, P. et al., Acta Chem. Scand. (A), 36(1982), 251.

[2] Burns, R. P., McCullough, F. P., Adtt. Inorg. Chem. Radiochem., 23(1977), 211.

[3] Yamaguchi, H., Kido, A., Uechi, T. et al., Bull. Chem. Soc. Jpn., 49(1976), 1271.

[4] Heinrich, D. D., Wang, J. C., Fackler, J. P., Acta Cryst., C46(1990), 1444.

[5] Lei, X. J., Huang, Z. Y., Liu, Q. T. et al., Inor g. Chem., 28(1989), 4302.

[6] Liu, H. Q., Cao, R., Lei, X. J. et al., J. Chem. Soc. Dalton Trans., 1990, 1023

[7] Huang, Z. Y., Lei, X. J., Kang, B. S. et al., Inorg. Chim. Act.., 169(1990), 25.

[ 8 ] Perreeaut, D. Druin, M., Michel. A. et al., Inorg. Chem., 30(1991), 3.

[ 9 ] Nagasundran, N., Ruper, G., Biscoe, J. et al., Inorg. Chem., 25(1986), 2947.

[10] Che, C. M., Wong, W. T., Lai, T. F. et al., J. Chem. Soc. Chem. Commun., 1983, 243. 